\title{
ERRATUM
}

\section{Neonatal perturbation of neurotrophic signaling results in abnormal sensorimotor gating and social interaction in adults: implication for epidermal growth factor in cognitive development}

T Futamura, A Kakita, M Tohmi, H Sotoyama, H Takahashi and H Nawa

Correction to: Molecular Psychiatry (2003) 8, 19-29. doi:10.1038/sj.mp.4001138

Molecular Psychiatry (2003) 8, 565. doi:10.1038/sj.mp.4001346

Table 1 from the above paper was missing. A copy of

the table is given below.

Table 1 The levels of monoamines and their metabolites in frontal cortex, striatum and brain stem of EGF and control rats

\begin{tabular}{|c|c|c|c|c|c|}
\hline & $D A$ & $D O P A C$ & $\begin{array}{c}\text { HVA } \\
\left(\mu g g^{-1} \text { wet tissue }\right)\end{array}$ & $5-H T$ & 5-HIAA \\
\hline \multicolumn{6}{|l|}{$\mathrm{P} 11$} \\
\hline \multicolumn{6}{|c|}{ Frontal cortex } \\
\hline Control & $0.038 \pm 0.002$ & $0.059 \pm 0.002$ & $0.159 \pm 0.004$ & $0.330 \pm 0.024$ & $0.272 \pm 0.038$ \\
\hline EGF & $0.037 \pm 0.005$ & $0.059 \pm 0.005$ & $0.153 \pm 0.011$ & $0.358 \pm 0.015$ & $0.282 \pm 0.028$ \\
\hline \multicolumn{6}{|l|}{ Striatum } \\
\hline Control & $5.521 \pm 0.189$ & $0.869 \pm 0.025$ & $0.720 \pm 0.029$ & $0.302 \pm 0.023$ & $0.577 \pm 0.053$ \\
\hline $\mathrm{EGF}$ & $5.834 \pm 0.137$ & $0.971 \pm 0.033^{*}$ & $0.730 \pm 0.024$ & $0.290 \pm 0.020$ & $0.627 \pm 0.085$ \\
\hline \multicolumn{6}{|l|}{ Brain stem } \\
\hline Control & $0.241 \pm 0.020$ & $0.141 \pm 0.006$ & $0.176 \pm 0.004$ & $0.239 \pm 0.106$ & $0.474 \pm 0.192$ \\
\hline EGF & $0.216 \pm 0.040$ & $0.140 \pm 0.017$ & $0.177 \pm 0.017$ & $0.157 \pm 0.087$ & $0.420 \pm 0.256$ \\
\hline \multicolumn{6}{|l|}{ P60 } \\
\hline \multicolumn{6}{|c|}{ Frontal cortex } \\
\hline Control & $0.210 \pm 0.009$ & $0.114 \pm 0.028$ & $0.095 \pm 0.012$ & $1.865 \pm 0.219$ & $0.709 \pm 0.132$ \\
\hline EGF & $0.228 \pm 0.028$ & $0.121 \pm 0.028$ & $0.111 \pm 0.012$ & $1.879 \pm 0.239$ & $0.774 \pm 0.159$ \\
\hline \multicolumn{6}{|l|}{ Striatum } \\
\hline Control & $15.007 \pm 0.804$ & $2.135 \pm 0.183$ & $1.400 \pm 0.067$ & $0.946 \pm 0.112$ & $1.012 \pm 0.185$ \\
\hline EGF & $14.730 \pm 0.771$ & $2.063 \pm 0.185$ & $1.395 \pm 0.088$ & $0.943 \pm 0.091$ & $1.026 \pm 0.191$ \\
\hline \multicolumn{6}{|l|}{ Brain stem } \\
\hline Control & $0.209 \pm 0.021$ & $0.509 \pm 0.065$ & $0.036 \pm 0.004$ & $0.633 \pm 0.027$ & $0.809 \pm 0.179$ \\
\hline EGF & $0.168 \pm 0.007$ & $0.327 \pm 0.013^{*}$ & $0.034 \pm 0.002$ & $0.570 \pm 0.052$ & $0.533 \pm 0.057$ \\
\hline
\end{tabular}

DA, dopamine; DOPAC, 3,4-dihydroxyphenylacetic acid; HVA, homovanillic acid; 5-HT, 5-hydroxytryptamine; 5-HIAA, 5 -hydroxyindoleacetic acid.

Monoamines and their metabolites were extracted from frontal cortex, striatum and brain stem at postnatal days 11 and 60 $(n=5)$. Their concentrations were determined by HPLC-ECD. ${ }^{*} P<0.05$. 